\title{
AUSLANDER-REITEN SEQUENCES AND GLOBAL DIMENSIONS
}

\author{
Wei Hu and Changchang XI*
}

\section{Introduction}

Almost split sequences were introduced by Auslander and Reiten. Their significant importance in the representation theory of Artin algebras and groups is well-known in the literature. In [11] the relationship between projective dimensions of modules appearing in an almost split sequence was studied. However, the relationship between the global dimensions of endomorphism algebras of these modules is unknown. In this paper we shall establish an inequality for global dimensions of these endomorphism algebras. This reveals also that almost split sequences may play a role in the study of representation dimensions of algebras, or the global dimensions of endomorphism algebras of certain modules. Our main result is

Theorem 1.1. Let $A$ be an Artin algebra, let $M$ be a generator-cogenerator for $A$ $\bmod$, and let $s$ be a positive integer. Then:

(1) If $\alpha_{i}: V_{i} \longrightarrow M_{i}^{\prime}$ is a left almost split morphism in A-mod with $M_{i}^{\prime}$ in add $(M)$ for $1 \leq i \leq s$, then gl.dim( $\left.\operatorname{End}_{A}\left(M \oplus \bigoplus_{i=1}^{s} V_{i}\right)\right) \leq \operatorname{gl} \cdot \operatorname{dim}\left(\operatorname{End}_{A}(M)\right)$.

(2) If $\beta_{i}: M_{i}^{\prime} \longrightarrow V_{i}$ is a right almost split morphism in $A$-mod with $M_{i}^{\prime}$ in add $(M)$ for $1 \leq i \leq s$, then gl.dim( $\left.\operatorname{End}_{A}\left(M \oplus \bigoplus_{i=1}^{s} V_{i}\right)\right) \leq \operatorname{gl} \cdot \operatorname{dim}\left(\operatorname{End}_{A}(M)\right)$.

As an immediate consequence we have

Corollary 1.2. Let $A$ be an Artin algebra and let $M$ be a generator-cogenerator for $A$-mod. If $0 \longrightarrow X \longrightarrow Y \longrightarrow Z \longrightarrow 0$ is an almost split sequence with $Y \in \operatorname{add}(M)$, then $\operatorname{gl} \cdot \operatorname{dim}\left(\operatorname{End}_{A}(M \oplus X \oplus Z)\right) \leq \max \left\{\operatorname{gl} \cdot \operatorname{dim}\left(\operatorname{End}_{A}(M \oplus X)\right), \operatorname{gl} \cdot \operatorname{dim}\left(\operatorname{End}_{A}(M \oplus\right.\right.$ $Z))\} \leq \operatorname{gl} \cdot \operatorname{dim}\left(\operatorname{End}_{A}(M)\right)$.

The proof of this result is given in Section 3 after we have made some preparations in Section 2. In Section 3 some corollaries of the main result are also discussed. The last section contains a few examples to illustrate the main result.

\section{Preliminaries}

In this section we recall some basic definitions and results needed in the paper.

Let $A$ be an Artin algebra, that is, $A$ is a finitely generated module over its center which is assumed to be a commutative Artin ring. We denote by $A$-mod (respectively,

Received by the editors November 26, 2005.

* Corresponding author. Fax: +86 10 58802136; Tel: 58808877.

2000 Mathematics Subject Classification: 16G70,16G10;18G20,16E30.

Keywords: Auslander-Reiten sequence, global dimension, coherent functor, generatorcogenerator. 
mod- $A$ ) the category of all finitely generated left (respectively, right) $A$-modules and by $\operatorname{rad}(A)$ the Jacobson radical of $A$. Given an $A$-module $M$, we denote by add $(X)$ the full subcategory of $A$-mod whose objects are summands of finite sums of copies of $X$. The projective dimension of $M$ is denoted by proj.dim $(M)$. The global dimension of $A$, denoted by gl.dim $(A)$, is the supremum of all proj.dim $(M)$ with $M \in A$-mod. The usual duality of an Artin algebra will be denoted by $D$. The composition of two morphisms $f: X \rightarrow Y$ and $g: Y \rightarrow Z$ between modules in $A$-mod will be denoted by $f g$.

Now let us recall some definitions from [7]. A morphism $f: X \longrightarrow Z$ in $A$-mod is said to be right almost split if (a) it is not a split epimorphism and (b) any morphism $X^{\prime} \longrightarrow Z$ which is not a split epimorphism factors through $f$. Dually, a morphism $g: Z \longrightarrow X$ is said to be left almost split if (a) it is not split monomorphism and (b) any morphism $Z \longrightarrow X^{\prime}$ which is not split monomorphism factors through $g$. Clearly, if a morphism $f: X \longrightarrow Z$ in $A$-mod is right almost split if and only if $D f: D(Z) \longrightarrow D(X)$ is left almost split in $A^{o p}$-mod, where we denote by $A^{o p}$ the opposite algebra of $A$.

A morphism $f: X \longrightarrow Y$ is called right minimal if any morphism $g: X \longrightarrow X$ with $g f=f$ is an automorphism. A morphism $f: X \longrightarrow Y$ is called minimal right almost split if it is both right minimal and right almost split. There is also an analogous concept of minimal left almost split morphism.

A short exact sequence $0 \longrightarrow X \stackrel{f}{\longrightarrow} Y \stackrel{g}{\longrightarrow} Z \longrightarrow 0$ in $A$-mod is called an almost split sequence if $f$ is a minimal left almost split morphism and $g$ is a minimal right almost split morphism. In many articles such a sequence is called an AR-sequence dedicated to Auslander and Reiten who first introduced and studied these sequences. Here we use the original language of Auslander and Reiten because the original terminology given by Auslander and Reiten reflects more transparently the properties of such exact sequences. Also, a minimal left (or right) almost spilt morphism was named as a source (or sink) map in some literature, we follow again the original terminology.

Let $\mathcal{C}$ be a full subcategory of $A$-mod and $X$ an arbitrary $A$-module. Recall from [8] that a morphism $f: C \longrightarrow X$ is called a right $\mathcal{C}$-approximation of $X$ if $C \in \mathcal{C}$ and the induced map $\operatorname{Hom}_{A}(-, f): \operatorname{Hom}_{A}\left(C^{\prime}, C\right) \longrightarrow \operatorname{Hom}_{A}\left(C^{\prime}, X\right)$ is surjective for all $C^{\prime} \in \mathcal{C}$. A minimal right $\mathcal{C}$-approximation of $X$ is a right $\mathcal{C}$-approximation of $X$ which is right minimal. Dually, there is the notion of a left $\mathcal{C}$-approximation and a minimal left $\mathcal{C}$-approximation.

Next, we recall the definition of representation dimension. For an Artin algebra $A$, Auslander in [3] defined the representation dimension of $A$, denoted by rep.dim $(A)$, as follows:

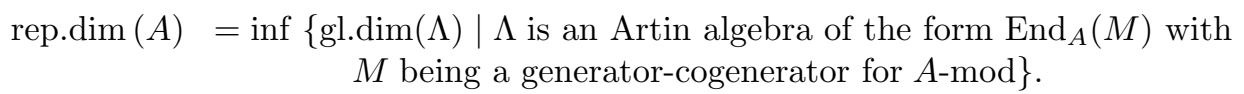

We say that an $A$-module $M$ is a generator (or cogenerator ) for $A$-mod if every $A$-module in $A$-mod is a quotient (or submodule) of a direct sum of copies of $M$.

If $M$ is a generator-cogenerator for $A$-mod with gl.dim( $\left.\operatorname{End}_{A}(M)\right)=\operatorname{rep} \cdot \operatorname{dim}(A)$, we say that the $A$-module $M$ defines the representation dimension of $A$. For more 
information on the representation dimension we refer to $[3,12,13]$ and the references therein.

For an additive category $\mathcal{C}$, we denote by $\widehat{\mathcal{C}}$ the category of coherent functors over $\mathcal{C}$. Recall from [1] that a functor $F: \mathcal{C}^{o p} \longrightarrow A b$ is coherent if there is an exact sequence $\left(-, C_{1}\right) \longrightarrow\left(-, C_{0}\right) \longrightarrow F \longrightarrow 0$ with $C_{i} \in \mathcal{C}$ for $i=0,1$. Here $A b$ is the category of all abelian groups, and $\left(-, C_{i}\right)$ stands for the functor $\operatorname{Hom}_{\mathcal{C}}\left(-, C_{i}\right)$. The relationship between coherent functors and module categories is as follows:

Lemma 2.1. Let $A$ be an Artin algebra and let $M$ be an A-module. Then

(1) $\operatorname{End}_{A}(M)-\bmod$ is equivalent to add $(M)$. In particular, gl.dim $\left(\operatorname{End}_{A}(M)\right)=$ gl.dim $(\widehat{\operatorname{add}(M)})$.

(2) Suppose that $M$ is a generator-cogenerator for $A$-mod, and that $m$ is a nonnegative integer. Then gl.dim( $\left.\operatorname{End}_{A}(M)\right) \leq m+2$ if and only if for each $A$-module $Y$ the coherent functor $\operatorname{Hom}_{A}(-, Y)$ has projective dimension at most $m$, that is, there is an exact sequence

$$
0 \rightarrow M_{m} \rightarrow \ldots \rightarrow M_{1} \rightarrow M_{0} \rightarrow Y \rightarrow 0,
$$

with $M_{j} \in \operatorname{add}(M)$ for $j=0, \ldots, m$, such that

$$
0 \rightarrow \operatorname{Hom}_{A}\left(-, M_{m}\right) \rightarrow \ldots \rightarrow \operatorname{Hom}_{A}\left(-, M_{1}\right) \rightarrow \operatorname{Hom}_{A}\left(-, M_{0}\right) \rightarrow \operatorname{Hom}_{A}(-, Y) \rightarrow 0
$$

is an exact sequence of functors on add $(M)$.

(3) If $A$ is not semisimple and if $M$ is a generator-cogenerator for $A$-mod, then gl.dim $\left(\operatorname{End}_{A}(M)\right)=2+\sup \left\{\right.$ proj.dim $\left.(-, X)\right|_{\operatorname{add}(M)} \mid X \in A$-mod $\}$, where $\left.(-, X)\right|_{\text {add }(M)}$ stands for the restriction of the functor $\operatorname{Hom}_{A}(-, X)$ to add $(M)$.

Proof. The statement (1) was proved by Auslander in [2], and the statement (2) was well-known from [3]. Let us prove (3).

Let $s=\sup \left\{\right.$ proj.dim $\left.(-, X)\right|_{\text {add(M) }} \mid X \in A$-mod $\}$. Clearly, it follows from (1) and (2) that gl.dim $\left(\operatorname{End}_{A}(M)\right) \leq 2+s$.

If $s$ is infinity, then gl.dim( $\left.\operatorname{End}_{A}(M)\right)$ is infinity by $(1)$ since $(-, X)$ is a coherent functor on $\operatorname{add}\left({ }_{A} M\right)$. In this case we have nothing to prove. So we may assume that $s$ is finite.

If $s=0$, then $\operatorname{add}(M)=A$-mod, that is, $A$ is representation-finite. Thus we have gl.dim( $\operatorname{End}(M))=2$ since $A$ is assumed to be non-semisimple. In this case, (3) is true.

Now we assume that $1 \leq s<\infty$. To prove that gl.dim( $\left.\operatorname{End}_{A}(M)\right)=2+s$, we take an indecomposable $A$-module $X$ which is not in $\operatorname{add}(M)$, and consider a minimal injective copresentation of $X$ :

$$
0 \longrightarrow X \stackrel{f}{\longrightarrow} I_{0} \stackrel{g}{\longrightarrow} I_{1}
$$

with $I_{i}$ injective for $i=0,1$. This provides an exact sequence of functors on $\operatorname{add}(M)$ :

$$
0 \longrightarrow(-, X) \longrightarrow\left(-, I_{0}\right) \longrightarrow\left(-, I_{1}\right) \stackrel{\pi}{\longrightarrow} F \longrightarrow 0,
$$

where $F$ is the cokernel of $\left(-, I_{0}\right) \longrightarrow\left(-, I_{1}\right)$. Note that the functor $\left(-, I_{i}\right)$ is projective-injective for $i=0,1$. If the map $\pi$ splits, then the kernel of $\pi$ is projectiveinjective. This implies that the functor $(-, X)$ on $\operatorname{add}(M)$ is projective; and thus $X$ lies in $\operatorname{add}(M)$, a contradiction. This shows that $\pi$ does not split. Similarly, we know that the map $(-, f)$ does not split. Hence the projective dimension of $F$ is 
proj.dim $\left.(-, X)\right|_{\text {add(M) }}+2$ since for a non-split exact sequence $0 \rightarrow K \rightarrow P \rightarrow G \rightarrow 0$ with $P$ projective it holds that proj.dim $(G)=\operatorname{proj} \cdot \operatorname{dim}(K)+1$. If we choose an indecomposable module $X$ such that proj.dim $\left.(-, X)\right|_{\operatorname{add}(\mathrm{M})}=s$, then the corresponding functor $F$ has projective dimension $s+2$. This finishes the proof.

Let us remark that the conclusion in Lemma 2.1(3) fails if $A$ is semi-simple, or if $M$ is just a generator, or a cogenerator for $A$-mod, but not the both. For the latter case, one may take an algebra of global dimension 2 and $M=A$ as a counterexample.

\section{Global dimensions of endomorphism algebras}

In this section we shall establish an inequality of the global dimensions of endomorphism algebras of modules that are generator-cogenerator, and that are linked somehow by an almost split sequence. It turns out that this can be used to reduce the global dimensions and produce many endomorphism algebras with the same global dimension.

The main result is the following theorem.

Theorem 3.1. Let $A$ be an Artin algebra and let $M$ be a generator-cogenerator for $A$-mod.

(1) If $\alpha: V \longrightarrow M^{\prime}$ is a left almost split morphism in $A$-mod with $M^{\prime}$ in $\operatorname{add}(M)$, then $\operatorname{gl} \cdot \operatorname{dim}\left(\operatorname{End}_{A}(M \oplus V)\right) \leq \operatorname{gl} \cdot \operatorname{dim}\left(\operatorname{End}_{A}(M)\right)$.

(2) If $\beta: M^{\prime} \longrightarrow V$ is a right almost split morphism in $A-\bmod$ with $M^{\prime}$ in add $(M)$, then gl.dim $\left(\operatorname{End}_{A}(M \oplus V)\right) \leq$ gl.dim $\left(\operatorname{End}_{A}(M)\right)$.

Proof. We may assume that $A$ is not semisimple. Let $\mathcal{C}=\operatorname{add}(M)$ and let $\mathcal{D}=$ $\operatorname{add}(M \oplus V)$. For any $A$-module $X$, we denote by $\operatorname{pd}_{\mathcal{C}}(-, X)$ the projective dimension of $\left.(-, X)\right|_{\mathcal{C}}$, and by $\operatorname{pd}_{\mathcal{D}}(-, X)$ the projective dimension of the functor $\left.(-, X)\right|_{\mathcal{D}}$. To prove the theorem, we shall use induction on $\operatorname{pd}_{\mathcal{C}}(-, X)$ to show that $\operatorname{pd}_{\mathcal{D}}(-, X) \leq$ $\operatorname{pd}_{\mathcal{C}}(-, X)$ for all $A$-modules $X$. Once this is done, our result follows from Lemma 2.1 (3) immediately.

Now we pick an arbitrary $A$-module $X$. If $\operatorname{gl} \cdot \operatorname{dim}\left(\operatorname{End}_{A}(M)\right)$ is infinite, there is nothing to prove. So we may assume that $\operatorname{gl} \cdot \operatorname{dim}\left(\operatorname{End}_{A}(M)\right)$ is finite.

If $\operatorname{pd}_{\mathcal{C}}(-, X)=0$, then $X$ is an object in $\mathcal{C}$, and hence in $\mathcal{D}$. $\operatorname{Thus}_{\mathcal{D}} \operatorname{pd}_{\mathcal{D}}(-, X)=0$. In this case we do have that $\operatorname{pd}_{\mathcal{D}}(-, X) \leq \operatorname{pd}_{\mathcal{C}}(-, X)$.

Now suppose that $\operatorname{pd}_{\mathcal{D}}(-, Y) \leq \operatorname{pd}_{\mathcal{C}}(-, Y)$ for all $A$-modules $Y$ with $\operatorname{pd}_{\mathcal{C}}(-, Y)<$ $s$, and we have to show that if $\operatorname{pd}_{\mathcal{C}}(-, X)=s>0$, then $\operatorname{pd}_{\mathcal{D}}(-, X) \leq \operatorname{pd}_{\mathcal{C}}(-, X)$. We may assume that $X$ is indecomposable.

If $X$ is isomorphic to $V$, then $\operatorname{pd}_{\mathcal{D}}(-, X)=0 \leq \operatorname{pd}_{\mathcal{C}}(-, X)$. So we may further assume that $X$ is not isomorphic to $V$. In this case, we take a minimal right $\mathcal{C}$ approximation $f: M_{0} \longrightarrow X$ of $X$ with $M_{0}$ in $\mathcal{C}$, and let $K$ be the kernel of $f$. Then we have an exact sequence

$$
0 \longrightarrow K \longrightarrow M_{0} \longrightarrow X \longrightarrow 0 .
$$

This induces the following exact sequence

$$
(*) \quad 0 \longrightarrow(-, K) \longrightarrow\left(-, M_{0}\right) \longrightarrow(-, X) \longrightarrow 0
$$

of functors on $\mathcal{C}$. Note that $\operatorname{pd}_{\mathcal{C}}(-, X)=\operatorname{pd}_{\mathcal{C}}(-, K)+1$. Hence $\operatorname{pd}_{\mathcal{D}}(-, K) \leq$ $\operatorname{pd}_{\mathcal{C}}(-, K)$ by induction hypothesis. In the following we show that $(*)$ is also exact on $\mathcal{D}$. It suffices to show that any morphism $g: V \longrightarrow X$ factors through $f$. Since 
$X$ is indecomposable and not isomorphic to $V$, the map $g$ is not a split monomorphism. Since $\alpha$ is a left almost split morphism, we know by definition that there is a homomorphism $g^{\prime}: M^{\prime} \longrightarrow X$ such that $g=\alpha g^{\prime}$. Since $M^{\prime} \in \mathcal{C}$ and since $f$ is a right $\mathcal{C}$-approximation, the map $g^{\prime}$ factors through $f$, and therefore $g$ factors through $f$. This implies that the sequence $(*)$ is exact on $\mathcal{D}$. Thus we have that $\operatorname{pd}_{\mathcal{D}}(-, X) \leq$ $\operatorname{pd}_{\mathcal{D}}(-, K)+1 \leq \operatorname{pd}_{\mathcal{C}}(-, K)+1=\operatorname{pd}_{\mathcal{C}}(-X)$, as desired.

For the proof of (2), we apply (1) to the left almost split morphism $D V \longrightarrow D\left(M^{\prime}\right)$ in $A^{o p}$-mod. Then we get that gl.dim $\left(\operatorname{End}_{A^{o p}}(D M \oplus D V)\right) \leq \operatorname{gl} \cdot \operatorname{dim}\left(\operatorname{End}_{A^{o p}}(D M)\right)$, that is, gl.dim $\left(\operatorname{End}_{A}(M \oplus V)^{o p}\right) \leq \operatorname{gl} \cdot \operatorname{dim}\left(\operatorname{End}_{A}(M)^{o p}\right)$. Since for any Artin algebra $A$ we have $\operatorname{gl} \cdot \operatorname{dim}(A)=\operatorname{gl} \cdot \operatorname{dim}\left(A^{o p}\right),(2)$ follows.

Before we state some consequences of Theorem 3.1, let us point out the following general formulation of Theorem 3.1.

Theorem 3.2. Let $A$ be an Artin algebra and let $M$ be a generator-cogenerator for $A$-mod.

(1) If $\alpha_{i}: V_{i} \longrightarrow M_{i}^{\prime}$ is a left almost split morphism in $A$-mod with $M_{i}^{\prime}$ in add $(M)$ for $1 \leq i \leq s$, then gl.dim( $\left.\operatorname{End}_{A}\left(M \oplus \bigoplus_{i=1}^{s} V_{i}\right)\right) \leq \operatorname{gl} \cdot \operatorname{dim}\left(\operatorname{End}_{A}(M)\right)$.

(2) If $\beta_{i}: M_{i}^{\prime} \longrightarrow V_{i}$ is a right almost split morphism in $A$-mod with $M_{i}^{\prime}$ in add $(M)$ for $1 \leq i \leq s$, then gl.dim $\left(\operatorname{End}_{A}\left(M \oplus \bigoplus_{i=1}^{s} V_{i}\right)\right) \leq \operatorname{gl} \cdot \operatorname{dim}\left(\operatorname{End}_{A}(M)\right)$.

A direct consequence of Theorem 3.1 is the following corollary.

Corollary 3.3. Let $A$ be an Artin algebra. If $X \longrightarrow Y$ is a left almost split morphism, or $Y \longrightarrow X$ is a right almost split morphism in A-mod, then, for any generatorcogenerator $M$ of $A$-mod, gl.dim $\left(\operatorname{End}_{A}(M \oplus X \oplus Y)\right) \leq \operatorname{gl.dim}\left(\operatorname{End}_{A}(M \oplus Y)\right)$.

As another immediate consequence of Theorem 3.1, we have the following result on the relationship between almost split sequences and global dimensions of endomorphism algebras.

Corollary 3.4. Let $A$ be an Artin algebra and let $M$ be an A-module which is a generator-cogenerator for $A$-mod. Suppose $0 \longrightarrow X \longrightarrow Y \longrightarrow Z \longrightarrow 0$ is an almost split sequence. Then gl.dim($\left(\operatorname{End}_{A}(M \oplus X \oplus Y \oplus Z)\right) \leq \operatorname{gl.dim}\left(\operatorname{End}_{A}(M \oplus Y)\right)$. In particular, if $Y$ is in $\operatorname{add}(M)$, then $\operatorname{gl} \cdot \operatorname{dim}\left(\operatorname{End}_{A}(M \oplus X \oplus Z)\right) \leq \operatorname{gl} \cdot \operatorname{dim}\left(\operatorname{End}_{A}(M)\right)$.

Proof. In the almost split sequence the map $X \longrightarrow Y$ is left almost split by definition. So we know from Theorem 3.1(1) that gl.dim $\left(\operatorname{End}_{A}(M \oplus X \oplus Y)\right) \leq$ gl.dim( $\left.\operatorname{End}_{A}(M \oplus Y)\right)$. Since $Y \longrightarrow Z$ is a right almost split morphism and since $Y$ lies in $\operatorname{add}(M \oplus X \oplus Y)$, it follows that gl.dim( $\left.\operatorname{End}_{A}(M \oplus X \oplus Y \oplus Z)\right) \leq \operatorname{gl} \cdot \operatorname{dim}\left(\operatorname{End}_{A}(M \oplus\right.$ $X \oplus Y))$ by Theorem 3.1(2). Thus gl.dim $\left(\operatorname{End}_{A}(M \oplus X \oplus Z)\right) \leq \operatorname{gl.dim}\left(\operatorname{End}_{A}(M)\right)$ since $Y$ lies in $\operatorname{add}(M)$.

As a special case of Corollary 3.4 we have the following result.

Corollary 3.5. (1) Let $A$ be an Artin algebra. If $V$ is a simple A-module and is not a composition factor for $\operatorname{rad}(I) / \operatorname{soc}(I)$ for any indecomposable non-simple injective $A$-module I, then gl.dim( $\left.\operatorname{End}_{A}(M \oplus \operatorname{Tr} D(V) \oplus V)\right) \leq \operatorname{gl} \cdot \operatorname{dim}\left(\operatorname{End}_{A}(M \oplus \operatorname{Tr} D(V))\right) \leq$ gl.dim( $\left.\operatorname{End}_{A}(M)\right)$, where $\operatorname{Tr} D$ is the inverse of the Auslander-Reiten translation DTr. 
(2) Let $A$ be an Artin algebra and let $S$ be a simple projective non-injective $A$ module. Then for any generator-cogenerator $M$ of $A$-mod, gl.dim $\left(\operatorname{End}_{A}(M \oplus \operatorname{Tr} D(S))\right.$ $\leq$ gl.dim( $\left.\operatorname{End}_{A}(M)\right)$.

Proof. (1) In this case we have an almost split sequence of the form

$$
0 \longrightarrow V \longrightarrow U \longrightarrow \operatorname{Tr} D(V) \longrightarrow 0
$$

with $U$ projective if $V$ is not injective. Thus (1) follows from Corollary 3.4.

(2) Under the assumption on $S$ there exists an almost split sequence

$$
0 \longrightarrow S \longrightarrow P \longrightarrow \operatorname{Tr} D(S) \longrightarrow 0,
$$

where $P$ is a projective $A$-module (see [4, proposition 1.17, p.13]). Thus the corollary follows by Theorem 3.1.

In the following we use $\Omega(M)$ to denote the first syzygy of a module $M$. For a self-injective algebra we have the following result.

Corollary 3.6. Let $A$ be a self-injective Artin algebra. Suppose $M$ is an A-module which is a generator-cogenerator for A-mod. If there is an almost split sequence $0 \longrightarrow X \longrightarrow Y \longrightarrow Z \longrightarrow 0$ in $A$-mod such that $\Omega(Y)$ lies in add $(M)$, then $\operatorname{gl} \cdot \operatorname{dim}\left(\operatorname{End}_{A}(M \oplus \Omega(X) \oplus \Omega(Z))\right) \leq \operatorname{gl} \cdot \operatorname{dim}\left(\operatorname{End}_{A}(M)\right)$.

Proof. For a self-injective algebra $A$, it follows from [5, proposition 5.1, p.14] that the given almost split sequence $0 \longrightarrow X \longrightarrow Y \longrightarrow Z \longrightarrow 0$ in $A$-mod induces an almost split sequence of the form $0 \longrightarrow \Omega(X) \longrightarrow \Omega\left(Y^{\prime}\right) \oplus P \longrightarrow \Omega(Z) \longrightarrow 0$, where $P$ is projective, and $Y^{\prime}$ is a direct summand of $Y$ and has no non-zero projective summand. Thus gl.dim $\left(\operatorname{End}_{A}(M \oplus \Omega(X) \oplus \Omega(Z))\right) \leq \operatorname{gl} \cdot \operatorname{dim}\left(\operatorname{End}_{A}(M)\right)$ by Corollary 3.4 .

Corollary 3.7. Let $A$ be an Artin algebra, $I$ an ideal in $A$, and $Z$ an indecomposable non-projective $A / I$-module. Suppose $0 \longrightarrow X \longrightarrow Y \longrightarrow Z \longrightarrow 0$ is an almost split sequence of $A$-modules and $M$ is a generator-cogenerator for $A / I$-mod. If $\operatorname{Hom}_{A}(A / I, Y)$ belongs to add $(M)$, then $\operatorname{gl} \cdot \operatorname{dim}\left(\operatorname{End}_{A / I}(M \oplus Z)\right) \leq \operatorname{gl} \cdot \operatorname{dim}\left(\operatorname{End}_{A / I}(M\right.$ $\left.\left.\oplus \operatorname{Hom}_{A}(A / I, Y)\right)\right)$.

Proof. This follows from the fact that the almost split sequence in $A$-mod induces an exact sequence $0 \longrightarrow \operatorname{Hom}_{A}(A / I, X) \longrightarrow \operatorname{Hom}_{A}(A / I, Y) \stackrel{g}{\longrightarrow} Z \longrightarrow 0$ with $g$ a right almost split morphism by [5, lemma 4.1].

Since for a generator-cogenerator $M$ the projective and injective modules are all contained in $\operatorname{add}(M)$, we would like to compare the global dimension of the endomorphism algebra of $M$ with that of the endomorphism algebra of $M \oplus V$, where $V$ is a module that can be constructed somehow from projective modules or injective modules. The following gives an partial answer.

Corollary 3.8. Let $A$ be an Artin algebra and let $M$ be an A-module which is a generator-cogenerator for A-mod.

(1) If there is a minimal left almost split morphism $V \longrightarrow U$ in $A$-mod with $V$ non-projective such that $D \operatorname{Tr}(U)$ lies in add $(M)$, then $\operatorname{gl} \cdot \operatorname{dim}\left(\operatorname{End}_{A}(M \oplus D \operatorname{Tr}(V))\right) \leq$ $\operatorname{gl.dim}\left(\operatorname{End}_{A}(M)\right)$. 
(2) If there is a minimal right almost split morphism $U \longrightarrow V$ in $A$-mod with $V$ non-injective such that $\operatorname{Tr} D(U)$ lies in add $(M)$, then $\operatorname{gld} \cdot \operatorname{dim}\left(\operatorname{End}_{A}(M \oplus \operatorname{Tr} D(V))\right) \leq$ gl.dim( $\left.\operatorname{End}_{A}(M)\right)$.

(3) If $P$ is an indecomposable projective non-injective $A$-module with $\operatorname{Tr} D(\operatorname{rad}(P))$ in add $(M)$, then $\operatorname{gl} \cdot \operatorname{dim}\left(\operatorname{End}_{A}(M \oplus \operatorname{Tr} D(P))\right) \leq \operatorname{gl} \cdot \operatorname{dim}\left(\operatorname{End}_{A}(M)\right)$.

(4) If $I$ is an indecomposable injective non-projective A-module with $D \operatorname{Tr}(I / \operatorname{soc}(I))$ in add $(M)$, then gl.dim $\left(\operatorname{End}_{A}(M \oplus D \operatorname{Tr}(I))\right) \leq \operatorname{gl} \cdot \operatorname{dim}\left(\operatorname{End}_{A}(M)\right)$.

Proof. It is well-known from [5, proposition 2.2] that the minimal left almost split morphism $V \longrightarrow U$ induces a minimal left almost split morphism of the form $D \operatorname{Tr}(V) \longrightarrow D \operatorname{Tr}(U) \oplus I^{\prime}$ with $I^{\prime}$ an injective $A$-module. Since $D \operatorname{Tr}(U)$ lies in $\operatorname{add}(M)$ and since $M$ is a generator-cogenerator for $A$-mod, we can apply Theorem 3.1 to the induced left almost split morphism and get the conclusion (1). (2) is a dual statement of (1).

As to (3), we note that in the given case we have a minimal right almost split morphism $Q \oplus \operatorname{Tr} D(\operatorname{rad}(P)) \longrightarrow \operatorname{Tr} D(P)$ with $Q$ a projective module. Thus the statement (3) follows from Theorem 3.1. (4) is a dual statement of (3).

Given a generator-cogenerator $M$ and a right almost split morphism $X \longrightarrow V$, sometimes it might happen that $X$ does not lie in $\operatorname{add}(M)$, while $\operatorname{Tr} D(X)$ might lie in $\operatorname{add}(M)$. In this case we have the following corollary.

Corollary 3.9. Let $A$ be an Artin algebra and let $M$ be a generator-cogenerator for $A$-mod.

(1) Suppose $f: X \longrightarrow V$ is a minimal right almost split morphism, where $V$ is not injective. If $\operatorname{Tr} D(X)$ lies in $\operatorname{add}(M)$, then $\operatorname{gl} \cdot \operatorname{dim}\left(\operatorname{End}_{A}(M \oplus V)\right) \leq \operatorname{gl} \cdot \operatorname{dim}\left(\operatorname{End}_{A}(M)\right)$.

(2) Suppose $f: V \longrightarrow X$ is a minimal left almost split morphism, where $V$ is not projective. If $D \operatorname{Tr}(X)$ lies in $\operatorname{add}(M)$, then gl.dim $\left(\operatorname{End}_{A}(M \oplus V)\right) \leq \operatorname{gl} \cdot \operatorname{dim}\left(\operatorname{End}_{A}(M)\right)$.

Proof. (1) By [5, proposition 2.3], for the given minimal right almost split morphism $f$ there is a minimal left almost split morphism of the form $V \longrightarrow \operatorname{Tr} D(X) \oplus P$ with $P$ a projective module. Thus we may apply Theorem 3.1 (1) to get the desired conclusion.

(2) follows dually because we have a minimal right almost split morphism $\mathrm{D} \operatorname{Tr}(X) \oplus$ $I \longrightarrow V$ with $I$ an injective $A$-module.

Next, we apply our results to stable equivalences. For a discussion of this subject with almost split sequence we refer to $[7$, Chapter $\mathrm{X}]$, and for stable equivalences of Morita type we refer to [10] and the references therein.

Corollary 3.10. Let $A$ and $B$ be two Artin algebras. Let $F: A$-mod $\longrightarrow B$-mod be an equivalence. Suppose $M$ is a generator-cogenerator for $A$-mod. Let $0 \longrightarrow$ $X \stackrel{f}{\longrightarrow} Y \oplus P \longrightarrow Z \longrightarrow 0$ be an almost split sequence in $A$-mod with $X, Y$ having no projective summands and $P$ projective. We assume further that $f$ is not zero in $A$-mod. Then if $Y \in \operatorname{add}(M)$, then gl.dimEnd $\operatorname{din}_{B}(F X \oplus F M \oplus D(B) \oplus B) \leq$ gl.dimEnd $_{B}(F M \oplus D(B) \oplus B)$.

Proof. Under the assumption we have an almost split sequence

$$
0 \longrightarrow F X \longrightarrow F Y \oplus Q \longrightarrow F Z \longrightarrow 0
$$

in $B$-mod by [6]. Thus the statement follows from Theorem 3.1. 
If we pass to the representation dimension, then we have the following statement.

Theorem 3.11. Let $A$ be an Artin algebra, and let $s$ be a positive integer. Then

(1) if $M$ defines the representation dimension of $A$ and if $V_{i} \longrightarrow M_{i}^{\prime}\left(\right.$ or $\left.M_{i}^{\prime} \longrightarrow V_{i}\right)$ is a left almost split morphism (or a right almost split morphism) in A-mod with $M_{i}^{\prime}$ in add $(M)$ for $1 \leq i \leq s$, then $M \oplus \bigoplus_{i=1}^{s} V_{i}$ defines the representation dimension of A.

(2) If $M$ defines the representation dimension of $A$ and if $0 \longrightarrow X \longrightarrow Y \longrightarrow$ $Z \longrightarrow 0$ is an almost split sequence in $A$-mod with $Y$ in add $(M)$, then $M \oplus X \oplus Z$ defines the representation dimension of $A$.

The following result is a consequence of Theorem 3.11 and a result in [9].

Corollary 3.12. Let $A$ be an Artin algebra with rep.dim $(A)=3$. If $M$ defines the representation dimension of $A$ and if $0 \longrightarrow X \longrightarrow Y \longrightarrow Z \longrightarrow 0$ is an almost split sequence in $A$-mod with $Y$ in add $(M)$, then, for any module $U \in \operatorname{add}(M \oplus X \oplus Z)$, the finitistic dimension of $\operatorname{End}_{A}(U)$ is finite.

Recall that the finitistic dimension of an Artin algebra $A$ is by definition the supremum of the projective dimensions of all finitely generated (left) $A$-modules of finite projective dimension. The finitistic dimension conjecture says that the finitistic dimension of any Artin algebra is finite, which still remains open. For some advances on the conjecture we refer to $[14,15]$ and the references therein.

Proof. It was shown in [9] that if an Artin algebra $\Gamma$ has global dimension at most three, then for any projective $\Gamma$-module $P$, the finitistic dimension of $\operatorname{End}_{\Gamma}(P)$ is finite. By Theorem 3.11, we know that the global dimension of $\operatorname{End}_{A}(M \oplus X \oplus Z)$ is at most three. So the corollary follows.

Remark. Our results in this paper can be formulated for any additive category with both an almost split sequence (or a left almost split morphism) and a generatorcogenerator. However, we would like to stick to the module categories of Artin algebras because this is our favorite subject.

\section{Examples}

In this section we display several examples to illustrate our main Theorem and the necessity of the conditions in our results.

Example 1. Suppose $A$ is an Artin algebra with a component of Auslander-Reiten quiver of the following form:

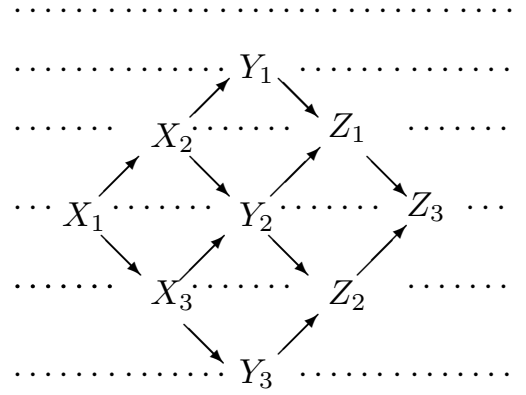


Then we know from Corollary 3.4 by putting $M=A \oplus D(A) \oplus \bigoplus_{i=1}^{3} Y_{i}$ that gl.dim $\left(\operatorname{End}\left({ }_{A}\left(A \oplus D(A) \oplus \bigoplus_{i=1}^{3}\left(X_{i} \oplus Y_{i} \oplus Z_{i}\right)\right)\right) \leq \operatorname{gl} \cdot \operatorname{dim}\left(\operatorname{End}_{A}\left(A \oplus D(A) \oplus \bigoplus_{i=1}^{3} Y_{i}\right)\right)\right.$.

Example 2. Let $k$ be an algebraically closed field of characteristic 2. We consider the group algebra $A=k A_{4}$ of the alternative group $A_{4}$. It is known that $A$ has 3 simple modules $k, \omega, \bar{\omega}$. Let $P(k), P(\omega)$ and $P(\bar{\omega})$ be the projective cover of these simple modules, respectively. Since group algebras are symmetric algebras, the Auslander-Reiten translation is the second syzygy. So the Auslander-Reiten quiver of $A$ has a component of the following form:

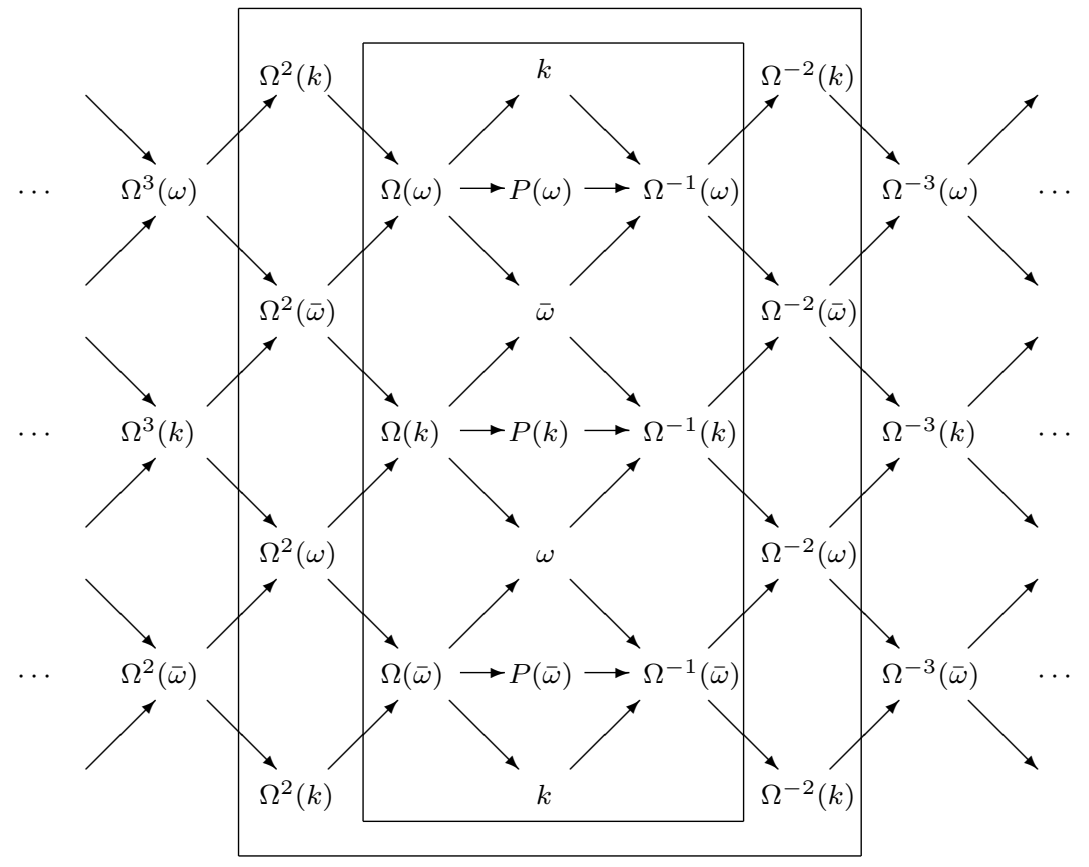

If we take $M$ to be the direct sum of the modules in the inner box, then we can easily get that the global dimension of $\operatorname{End}_{A}(M)$ is 3. By Corollary 3.4, the global dimension of the endomorphism algebra of the direct sum of the modules insider the out box is also 3 .

Example 3. Let $A=k[x, y] /\left(x^{2}, y^{2}\right)$, where $k$ is an arbitrary field. Then $A$ is a symmetric algebra, and the Auslander-Reiten translation is just the second syzygy $\Omega^{2}$. So the Auslander-Reiten quiver of $A$ has a component of the following form:

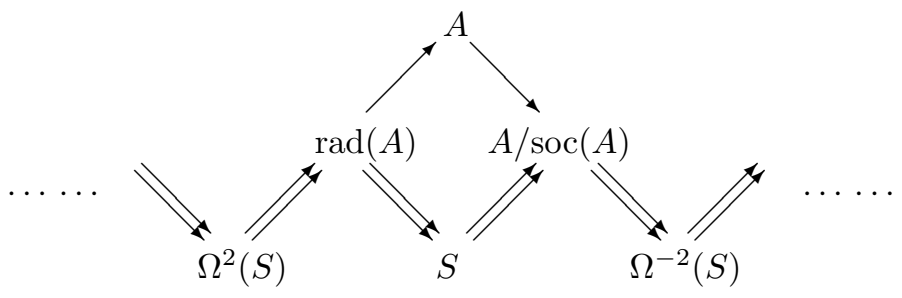


Let $M=A \oplus \operatorname{rad}(A) \oplus A / \operatorname{soc}(A) \oplus S$. A computation shows that gl.dim $\left(\operatorname{End}_{A}(M)\right)$ $=3=\operatorname{rep} \cdot \operatorname{dim}(A)$. Thus for any $i, j \in \mathbb{Z}$, gl.dim $\left(\operatorname{End}_{A}\left(M \oplus \bigoplus_{j=1}^{i} \Omega^{2 j}(S)\right)\right)=3$ by Theorem 3.11.

Example 4. The condition that $M$ is a generator-cogenerator in Theorem 3.1 can not be weakened to that $M$ is just a generator or a cogenerator for $A$-mod. For example, we consider the algebra $A$ given by quiver

$$
1 \stackrel{\alpha}{\longleftarrow} 2 \stackrel{\beta}{\longleftarrow} 3
$$

with the relation $\beta \alpha=0$. We denote by $P(i)$ and $S(i)$ the indecomposable projective module and the simple module corresponding to the vertex $i$, respectively. For this algebra $A$ there is an almost split sequence

$$
0 \longrightarrow S(2) \longrightarrow P(3) \longrightarrow S(3) \longrightarrow 0 \text {. }
$$

Thus we have a right almost split morphism $P(3) \longrightarrow S(3)$. If we take $M=A$, then gl.dim$\left(\operatorname{End}_{A}(M)\right)=2$. However, if we consider the endomorphism algebra of ${ }_{A} M \oplus S(3)$, then it is given by the quiver

$$
1 \stackrel{\alpha}{\longleftarrow} 2 \stackrel{\beta}{\longleftarrow} 3 \stackrel{\gamma}{\longleftarrow} 4
$$

with the relations $\beta \alpha=\gamma \beta=0$, and $\operatorname{gl} \cdot \operatorname{dim}\left(\operatorname{End}_{A}(M \oplus S(3))\right)=3$. This shows that the condition that $M$ is a generator-cogenerator in Theorem 3.1 can not be replaced by a generator. Similarly, we may take $M^{\prime}=D\left(A_{A}\right)$ and consider the left almost split morphism $S(1) \longrightarrow P(2)$. Clearly, we get that the global dimension of $\operatorname{End}_{A}\left(M^{\prime}\right)$ is 2 and the global dimension of $\operatorname{End}_{A}\left(M^{\prime} \oplus S(1)\right.$ is 3 . This shows that the condition that $M$ is a generator-cogenerator in Theorem 3.1 can not be weakened to that $M$ is a cogenerator.

Example 5. The condition of left (right) almost split morphisms in Theorem 3.1 cannot be weakened to irreducible morphisms. This can be shown by the following example. Let $A$ be an algebra given by the quiver

$$
1 \stackrel{\alpha}{\longleftarrow} 2 \stackrel{\beta}{\longleftarrow} 3 \stackrel{\gamma}{\longleftarrow} 4
$$

Then we have an irreducible morphism from $I(3) / \operatorname{soc}(I(3))$ to $I(2)$, where $I(i)$ stands for the indecomposable injective module corresponding to the vertex $i$ in the quiver. Let $M$ be the direct sum of all indecomposable projective modules and all indecomposable injective modules. We consider the endomorphism algebras of $M$ and $M \oplus I(3) / \operatorname{soc}(I(3))$. One can verify that the algebra $\Lambda:=\operatorname{End}_{A}(M)$ is given by the quiver

$$
1 \stackrel{\alpha}{\longleftarrow} 2 \stackrel{\beta}{\longleftarrow} 3 \stackrel{\gamma}{\longleftarrow} 4 \stackrel{\delta}{\longleftarrow} 5 \stackrel{\eta}{\longleftarrow} 6 \stackrel{\epsilon}{\longleftarrow} 7,
$$

with relations $\delta \gamma \beta \alpha=\eta \delta \gamma \beta=\epsilon \eta \delta \gamma=0$, and that the algebra $\Gamma:=\operatorname{End}_{A}(M \oplus$ $I(3) / \operatorname{soc}(I(3)))$ is given by the quiver

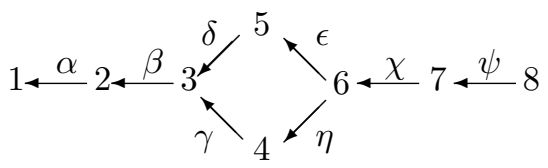


with the relations $\psi \chi \epsilon=\chi \eta \gamma \beta=\epsilon \delta-\eta \gamma=\delta \beta \alpha=0$. A calculation shows that gl.dim $(\Lambda)=3$ and gl.dim $(\Gamma)=4$. This implies that even for a generator-cogenerator the left (right) almost split morphism in Theorem 3.1 cannot be replaced by an irreducible morphism.

\section{Acknowledgements}

The authors acknowledge gratefully the support from the Cultivation Fund of the Key Scientific and Technical Innovation Project (No. 704004), and the Doctor Program Foundation (No. 20040027002), Ministry of Education of China; and the "985 Program" of the Beijing Normal University under the project "Representation Theory and Related Topics". Also, the support from the Alexander von Humboldt Foundation is greatly appreciated.

\section{References}

[1] M. Auslander, Coherent functors. Coherent functors. 1966 Proc. Conf. Categorical Algebra (La Jolla, Calif., 1965) pp. 189-231 Springer, New York.

[2] _ _ Representation theory of Artin algebras I. Comm. Algebra 1 (1974) 177-268.

[3] _ Representation dimension of Artin algebras. Queen Mary College Mathematics Notes, Queen Mary College, London, 1971.

[4] M. Auslander, M. I. Platzeck, and I. Reiten, Coxeter functors without diagrams, Trans. Amer. Math. Soc. 250 (1979) 1-46.

[5] M. Auslander and I. Reiten, Representation theory of Artin algebras V: Methods for computing almost split sequences and irreducible morphisms. Comm. Algebra 5 (1977) 519-554.

[6] __ Representation theory of Artin algebras VI: A functorial approach to almost split sequences, Comm. Algebra 6 (1978) 257-300.

[7] M. Auslander, I. Reiten, and S. Smalø, Representation theory of Artin algebras. Cambridge Studies in Advanced Mathematics 36, Cambridge University Press, 1995.

[8] M. Auslander and S .O. Smalø, Preprojective modules over Artin algebras, J. Algebra 66 (1980) $61-122$.

[9] K. Igusa and G. Todorov, On the finitistic global dimension conjecture for artin algebras. In: Representations of algebras and related topics, 201-204, Fields Inst. Commun. 45, Amer. Math. Soc., Providence, RI, 2005.

[10] Y. M .Liu and C. C. Xi, Constructions of stable equivalences of Morita type for finite-dimensional algebras III, preprint at http://math.bnu.edu.cn/ ccxi/.

[11] D. Madsen, Projective dimensions and almost split sequences, J. Algebra 271 (2004) 652-672.

[12] C. C. Xi, Representation dimension and quasi-hereditary algebras, Adv. Math. 168 (2002), no. 2, 193-212.

[13] _ Adjoint functors and representation dimensions, Acta Math. Sin. (Engl. Ser.) 22 (2006), no. 2, 625-640.

[14] _ On the finitistic dimension conjecture II: related to finite global dimension, Adv. Math. 201 (2006) 116-142.

[15] _ On the finitistic dimension conjecture III: related to the pair eAe $\subseteq$ A. Preprint at http://math.bnu.edu.cn/ ccxi/.

School of Mathematical Sciences, Beijing Normal University, 100875 Beijing, People's Republic of China

E-mail address: hwxbest@163.com, xicc@bnu.edu.cn 